the size of the glass globe has been properly judged, it will stay in because a broad and continuous surface of contact is secured between the lips of the wound.

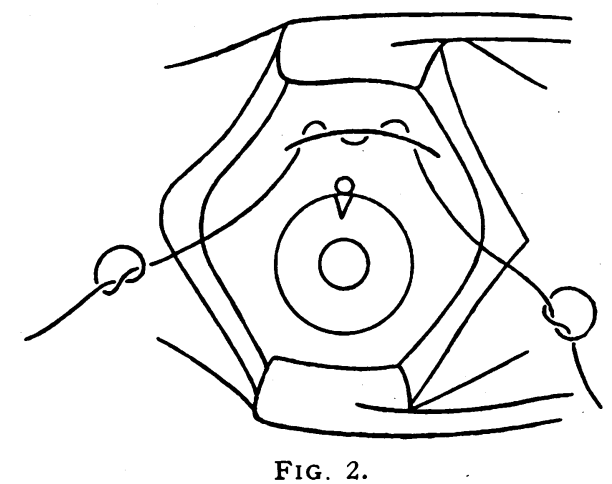

Horse hair suture for closing the conjunctival wound after trephining.

The same suture may be used with advantage for closing the wound in the conjunctiva after trephining. It is passed in and out as shown in Fig. 2, and, as in the previous operation, it is easily removed. No difficult silk knots to find and cut! No cocaine necessary to assist in their removal.

\title{
THE PATHOLOGY OF SCLERAL PLAQUES \\ Report of five cases of degenerative plaques in the sclera mesially, one studied histologically
}

BY

Arthur M. Culler, M.D.

DAYTON, OHIO

UNDER the heading, "Bilateral mesial superficial deficiency of the sclera," Basil Graves ${ }^{1,2}$ has recently described two cases of this disease. I have encountered this condition (which on casual observation I thought might be a hyaline change in the insertion of the internal rectus muscle) and found that the edge of the lesion lay approximately $3 \mathrm{~mm}$. behind the mesial conjunctival limbus. It presented the appearance under the slit-lamp of a minute squared hole in the outer layers of the sclera through which the underlying ciliary body could be seen. A slightly congested conjunctiva was neither elevated nor depressed over the lesion. 
The area was of the same slate gray colour as a scleral staphyloma but the borders were sharply demarcated, giving a punched out appearance. There is nothing to add to Graves' accurate description. I have fortunately obtained an autopsy specimen of the lesion.

Case I.-M.C., a female, aged 79 years, was seen in consultation with the Medical Department of Good Samaritan Hospital. Her fundus showed well marked arteriosclerosis of the retinal vessels, and incipient cataract was present. Externally there was a broad gerontoxon completely encircling the cornea. In each eye was a dark area in the sclera which has been described above. The m easurements of the areas were as follows: O.D. conjunctival limbus to mesial border of the lesion $322 \mathrm{~mm}$.; vertical mean diameter $5.4 \mathrm{~mm}$.; horizontal mean diameter $2.0 \mathrm{~mm}$.; O.S. limbus to mesial border $2.9 \mathrm{~mm}$.; vertical mean diameter $3.3 \mathrm{~mm}$.; horizontal mean diameter $1.3 \mathrm{~mm}$.

The patient succumbed after a long illness and permission was obtained for a scleral specimen at autopsy. The important autopsy findings were as follows : arteriosclerotic heart and kidney disease, cachexia, and terminal pneumonia.

A specimen approximately $6 \mathrm{~mm}$. in diameter was removed from the mesial aspect of the sclera of the right eye. The apparent hole, $5.5 \mathrm{~mm}$. by $2.0 \mathrm{~mm}$. was filled with a transparent hardened seed-like mass which exhibited a marked tendency to break away from the surrounding sclera and could have been shelled out. Fat stains were not done. The specimen was fixed in 10 per cent. formalin, and imbedded in paraffin. Serial sections were stained with haematoxylin and eosin or Cajal's connective tissue stain.

In the sections the conjunctiva and episclera were atrophic. A few globules filled with granular debris were seen lying immediately under the epithelium. The sclera was $0.6 \mathrm{~mm}$. in thickness at the edge of the specimen but became gradually thinner as the lesion was approached. The borders of the lesion were slightly elevated, perhaps largely due to artefact, but the sclera in the centre of the lesion was only $0.3 \mathrm{~mm}$. in thickness. The lesion itself was sharply demarcated. The temporal border (towards the cornea) (Fig. 1) dipped into the sclera in a gradual curve (Fig. 2), until in the centre of the lesion the strand of sclera forming the base was less than one-tenth the thickness of the lesion (Fig. 3). The mesial border was more nearly perpendicular (Fig. 4). The lesion stained deeply. It was composed of a large mass of granular debris which was entirely free from cellular structure and contained large masses of hyaline. No clefts or vacuoles could be made out either in the mass or in the surrounding sclera which might have resulted from the presence of lipoid degenerative changes. There were no calcium deposits. 
Surrounding the degenerative plaque was a zone approximately $0.5 \mathrm{~mm}$. broad in which the connective tissue fibres appeared swollen, and the nuclei for the most part had disappeared. Individual fibres could be made out passing abruptly into the mass of hyaline.

Case II.-L.S., a female, aged 79 years, presented herself on December 13, 1937, because of burning of her eyelids. She was refracted, obtaining 6/7.5 vision in both eyes. Ophthalmoscopic examination showed some sclerosis of the nucleus and a few riders in the lens of each eye, and well marked tortuosity and variation in the calibre of the arteries. There was a moderate hypertrophic conjunctivitis. The lids were flaccid. There was a prominent gerontoxon and slate gray, roughly rectangular areas were present in the sclera mesially in both eyes. The borders of the lesions were quite sharp although this was variable (Fig. 5). The measurements were as follows: O.D. limbus to mesial border of the plaque $3.7 \mathrm{~mm}$.; vertical mean diameter $3.3 \mathrm{~mm}$.; horizontal mean diameter $1.0 \mathrm{~mm}$.; O.S. limbus to lesion $3.5 \mathrm{~mm}$.; vertical mean diameter $2.5 \mathrm{~mm}$.; horizontal diameter $1.3 \mathrm{~mm}$.

Case III.-G.T., a female, aged 85 years, presented herself with a contact dermatitis of the lids on April 16, 1938. Her corrected vision was O.D. 6/7.5, O.S. 6/12. Ophthalmoscopic examination revealed scattered flaky opacities in the cortex of the lens and exaggerated arteriosclerosis of the retinal vessels in both eyes. There were broad circular gerontoxons in both eyes and each presented a slate gray area in the sclera mesially. The right was much broader below than above and the borders were less sharp. The left lesion was narrow in the centre so that it appeared two-lobed (Fig. 6). O.D. conjunctival limbus to mesial border of the lesion $3.0 \mathrm{~mm}$.; vertical measurement $5.0 \mathrm{~mm}$.; horizontal $2.0 \mathrm{~mm}$. O.S. limbus to lesion $3.9 \mathrm{~mm}$.; vertical measurement $4.2 \mathrm{~mm}$.; horizontal diameter $21 \mathrm{~mm}$.

Case IV.-M.H., a female, aged 82 years, consulted me on November 1, 1937, because of failing vision in both eyes. She had moderately contracted visual fields. Her corrected vision was $6 / 60$ in both eyes. The fundus showed some cupping of the disc and incipient cataracts in both eyes. Intra-ocular tension was O.D. 35, O.S. 32 (Schiötz). External examination showed only a slight arcus senilis. In the mesial aspect of the sclera of the left eye there was an oval, punched out dark area. The mesial border was $3.1 \mathrm{~mm}$. from the limbus. The vertical diameter was $1.2 \mathrm{~mm}$. The horizontal diameter was $0.9 \mathrm{~mm}$. The right eye showed no well defined dark area, although under the slitlamp superficial changes in the sclera could be made out.

Case V.-D.F., a male, aged 72 years, complained on September 7,1937 , of occasional attacks of blurred vision during which 


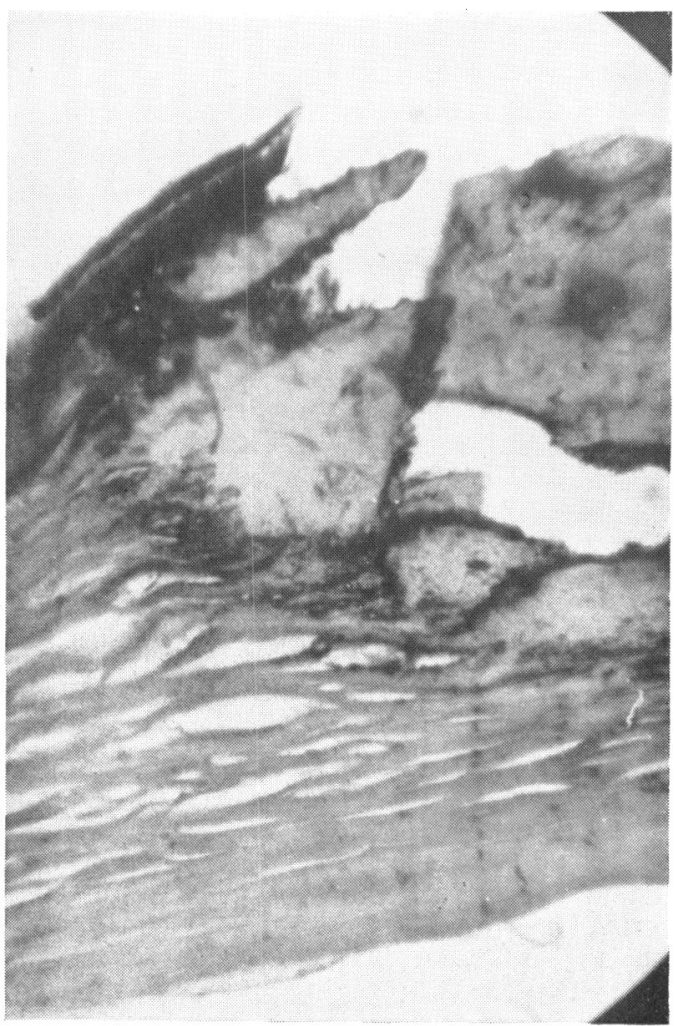

FIG. 1.

Photomicrograph $\times 150$. Section through the left border of the lesion from the right eye of Case 1. Swollen fibres of the sclera can be seen passing into a friable mass of cellular debris. 


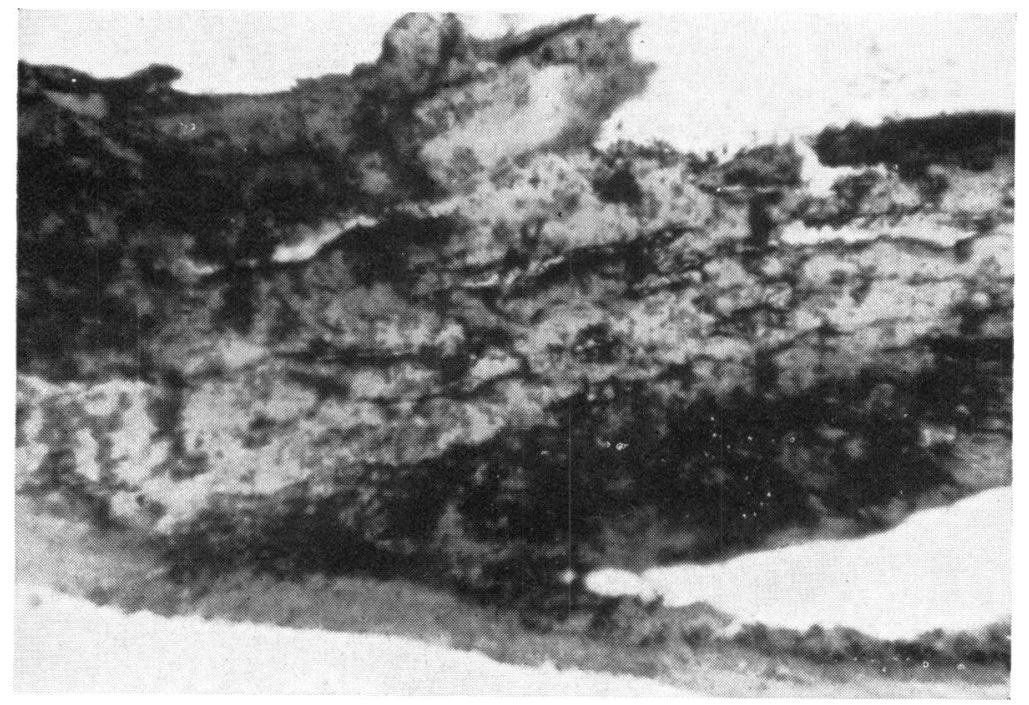

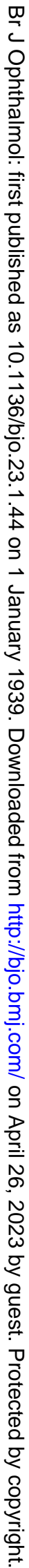

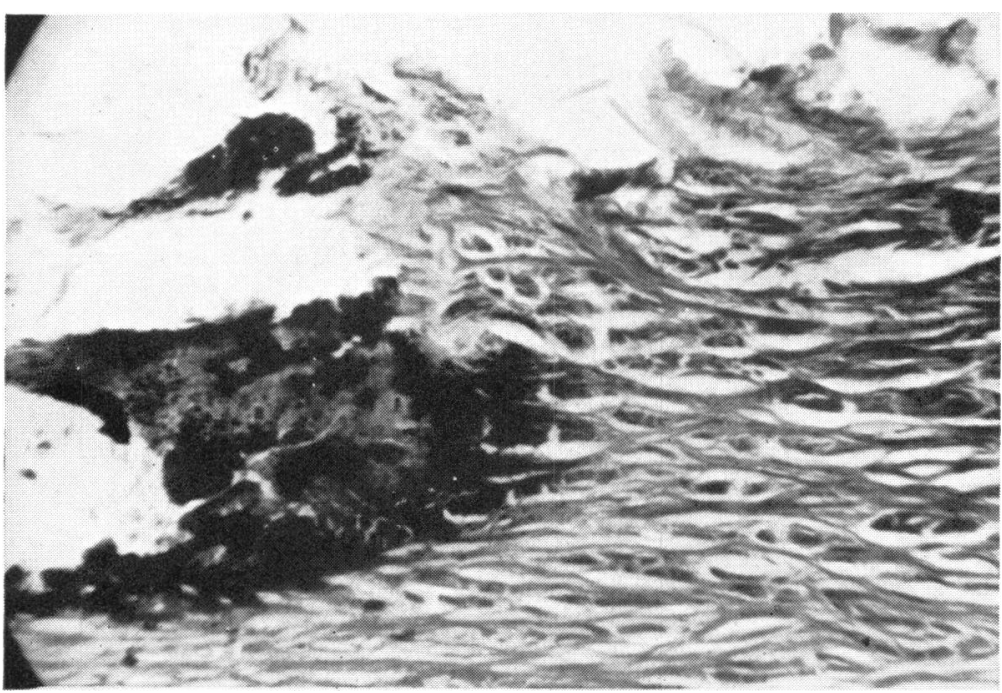

FIG. 4.

The right border of the lesion in the same section. This border is more nearly perpendicular. The hyaline mass has broken away from the sclera in the superficial portion. (×150.) 


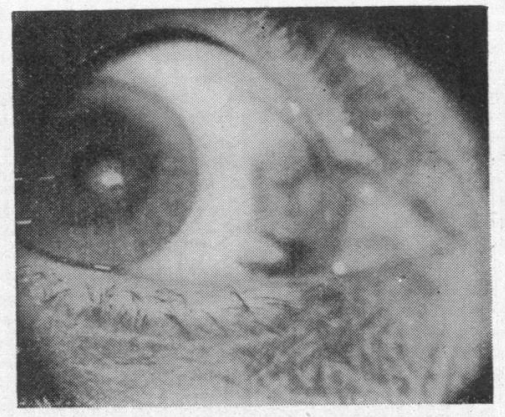

FIG. 5.

Photograph $(\times 1.5)$ of the right eye of Case 2 , showing the slate gray rectangular plaque in the sclera mesially.

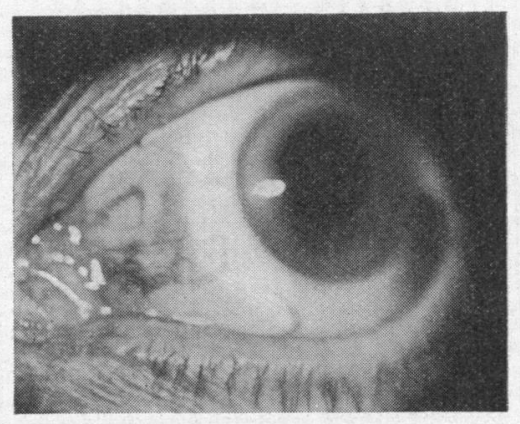

Fig. 6.

Photograph of the left eye of Case 3, showing the two lobed lesion in the sclera mesially Con junctival vessels dilated by the light necessary for the photograph partially obscure the lesion.

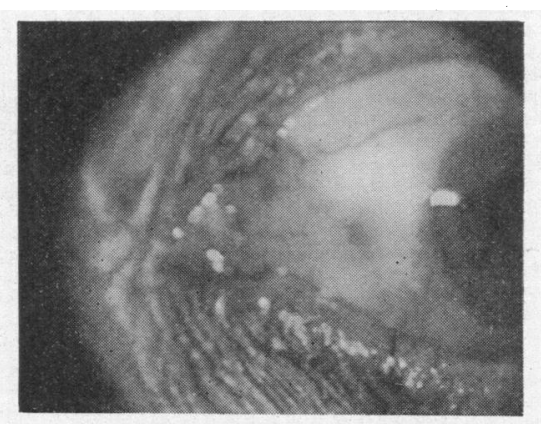

Photograph of the left eye of Case 4, showing the dark oval spot in the mesial aspect of the sclera. 


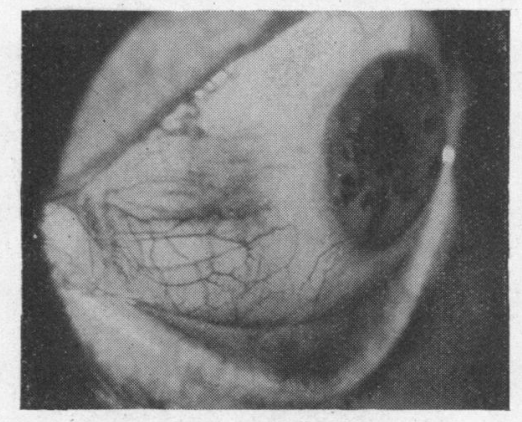

FIG. 8 .

Photograph of the left eye of Case 5, showing the rectangular defect in the sclera. This has less sharp borders than the other cases but is more conspicuous clinically than in the photograph because of the colour contrast.

he saw halos around lights. No flashes of light and no pain were apparent. His right eye had been enucleated at the age of 16 years, because of trauma. His visual acuity in the left eye was $6 / 30$ corrected to $6 / 7.5$. The fundus showed shallow cupping of the disc and a posterior cortical radiating opacity at " 1.30 o'clock." Visual fields showed a sickle-shaped scotoma above and below the blind spot. Intra-ocular tension was 45 (Schiötz). Externally there was a well marked arcus senilis and a large rectangular dark area in the sclera mesially. The borders of the lesion were everywhere less abrupt than in the other cases. The measurements were as follows : limbus to lesion $42 \mathrm{~mm}$.; vertical diameter of lesion $5.2 \mathrm{~mm}$; horizontal diameter $2.6 \mathrm{~mm}$.

\section{Comment}

While these lesions are small, they are rather conspicuous clinically because of the colour. Photographs do not show them well because of scleral scatter. Under the slit-lamp the borders of the lesions are usually sharp and in places slightly shelved. This was obscured by the dispersion of light in all of my attempts at slit-lamp photographs.

The two cases described in detail by Graves were respectively 60 and 70 years of age. The cases described here were all over 70 . One might expect degenerative lesions to occur in this age group more frequently than in those under 60 years of age. The histclogical appearance is clearly a degenerative change.

Rones $^{3}$ in a comprehensive survey of senile changes in the 
eye does not mention this condition and states that senile changes in the sclera are chiefly in the nature of fatty infiltration.

Graves mentions having seen six or seven cases of this disease. Probably it is not at all rare but has failed to appear in the literature previously because it is apparently clinically insignificant. However, the photomicrographs of the case studied histologically show how near the sclera is to perforation.

Van der Hoeve ${ }^{4}$ has described an entity, scleromalacia perforans, which arises in degenerative plaques in the sclera. The question arises whether Graves' scleral spots are not the early stage of the same disease.

Verhoeff and King ${ }^{5}$ in a study of the literature from which they have collected fourteen cases, and of their own case, have composed a description of the clinical course of scleromalacia perforans. I quote from their paper. "In one or both eyes there develop simultaneously or at intervals, slightly elevated nodules involving the sclera and overlying tissue and situated anywhere between the corneal limbus and equator. Congestion is moderate and is limited to the nodules and their immediate vicinities. After a considerable period of time, in some cases six months or longer, one or more of the nodules may disappear. At the former site of the nodule there is seen in the sclera a shallow cavity, often of large size. Over a cavity the conjunctiva may be intact or absent. When, as rarely happens, a lesion is situated at the corneal limbus, it may perforate into the anterior chamber, and produce a cystoid nodule such as occurs after trephining. Into this nodule, the iris may or may not be herniated. When situated elsewhere, the lesion seldom if ever perforates the sclera and the remaining tissue usually withstands the intraocular pressure, rupturing only in rare instances. Evidences of intraocular inflammation are relatively slight or entirely lacking, unless as rarely happens, purulent infection occurs at the site of a cavity. Pain may be absent.

"Posterior synechiae, atrophy of the iris, or cataract, may occur as late complications in marked cases. Usually the ocular disease does not occur before the patient reaches the age of fifty years. In the great majority of cases definite evidences of rheumatoid arthritis exist at the onset of scleritis."

From the histological findings in their case they believe the process is comparable to the subcutaneous nodules of rheumatoid arthritis. They postulate a metabolic disturbance which produces a deposition of some chemical substance in the sclera. A sharply defined area of necrotic tissue appears. "This becomes surrounded by a wall of epithelioid cells and slowly infiltrated with pus cells. This process .results in the formation of a sequestrum which becomes completely disintegrated and densely infiltrated 
with necrotic pus cells." Verhoeff believes that cavities are due to perforation and discharge of the sequestrum, though the necrotic tissue is sometimes replaced by fibrous tissue without cavity formation.

There are obvious differences in the clinical picture of these tiny degenerative plaques and scleromalacia perforans. None of my patients suffered from serious arthritis. In none were nodules ever present on the sclera. On the other hand, these lesions have not been described in any other location than between the insertion of the internal rectus and the limbus, whereas the nodules of the early stage of scleromalacia perforans may occur anywhere in the sclera which is covered with conjunctiva. These differences are irreconcilable. One must conclude that recent literature has added two new lesions of the sclera, both primarily degenerative in character.

\section{Summary}

Graves' scleral plaques occur principally in patients over sixty years of age. Approximately $3 \mathrm{~mm}$. behind the corneal limbus mesially, irregularly rectangular translucent plaques in the superficial layers of the sclera may occur. These vary in size from pinpoint to approximately $2 \mathrm{~mm}$. in the horizontal plane and $6 \mathrm{~mm}$. in the vertical plane and are usually bilateral. They are slate gray in colour because of the ciliary body showing through the area of increased transparency. They do not grossly disturb the neighbouring conjunctiva or ciliary body. A distinguishing characteristic is the definite tendency to square cut borders. To the descriptive name applied by Graves, "Developmental bilateral mesial superficial intagliated deficiency of the sclera," can only be added that the histological appearance is that of a degenerative change' rather than a developmental defect. No clinical symptoms are known to arise from the lesions. Scleromalacia perforans does nọt arise in these degenerative plaques in the sclera.

\section{BIBLIOGRAPHY}

1. Graves, Basil.-Bilateral mesial superficial deficiency of the sclera. Brit. Jl. of Ophthal., Vol. XXI, No. 10, p. 534, October, 1937.

2. ' Diseases of the Cornea. "The Eye and its Diseases.". Berens, pp. 467-8. (Saunders, 1936.)

3. Rones, BENJ.- Senile changes and degenerations of the human eye: Amer. Jl. of Ojhthal., Vol. XXI, No. 3, pp. 243-4, March, 1938.

4. VAN DER Hoeve, J.-Scleromalacia Perforans. Arch. of Ophthal., Vol. XI, p. 111, January, 1934.

5. Verhoeff, F. H., and KING, M. J.-Scleromalacia Perforans: Report of a case studied clinically, bacteriologically and histologically. Trans. of the Section of Ophthal., 1938. (In'Press.) 